Background: Treatment response for melancholic patients may differ from nonmelancholic depressed patients, with broader spectrum antidepressant drugs being required for the melancholic subtype. Outpatients $(n=202)$ meeting the diagnostic criteria for major depressive disorder (DSM-IV), with HAMD17 $\geq 20$ and melancholic features, participated in an 8-week, multicenter, open-label, naturalistic, variable dose pilot study (F1J-AY-HMCZ).

Method: All patients received duloxetine $60 \mathrm{mg} /$ day for the first 4 weeks. In patients not responding by this time, effectiveness of duloxetine treatment was compared between patients who either continued on $60 \mathrm{mg}$ or received a higher, flexible $(90-120 \mathrm{mg} /$ day) dose, for a second 4-week period.

Results: After the initial 4-week period, $40.6 \%$ of patients $(n=82)$ responded $(\geq 50 \%$ reduction from baseline HAMD17) to duloxetine $60 \mathrm{mg} /$ day, and $17.8 \%(n=36)$ achieved formal remission (HAMD17 $\leq 7)$ of symptoms. For nonresponders at 4 weeks, no significant differences were detected across the $60-\mathrm{mg}$ and 90 - to $120-\mathrm{mg}$ treatment groups in the rates of response $(47.9 \%$ and $42.9 \%$, respectively) or remission $(22.9 \%$ and $18.4 \%)$ over the next 4 weeks. Overall, $58 \%$ of enrolled patients responded to treatment by study completion. Nausea, headache, dry mouth and constipation were the most frequently reported emergent adverse events.

Conclusions: The overall response rates after 4 and 8 weeks were encouraging for this sample of melancholic patients. In patients who did not respond to duloxetine $60 \mathrm{mg}$ over the first 4 weeks of treatment, increasing to a higher dose in the second month was not associated with any greater clinical benefit.

\section{Censoring of prescribable doses: the case of risperidone long-acting injection}

\section{T Lambert}

OPEN/ORYGEN, University of Melbourne, Melbourne, Australia

Background: Suggested dose ranges for new medicines often prove to be different from those that are empirically derived in the field. The release of the first long-acting SGA (risperidone CONSTA) suggests that recommended doses are, in the main, too low and the highest dose does not allow the full spectrum of doses that are seen with the oral equivalent. To investigate this, recommended CONSTA doses (in risperidone oral equivalents) are compared with the distribution of doses found in a large pharmacoepidemiological database.
Methods: From the OPEN/SEER pharmacoepidemiological data archives. Mean doses of SGAs are examined over time and most likely mean stable doses calculated. These are compared with projected mean doses and are further compared with suggestions based on the Seeman's radioligand-free Kis. CONSTA is examined in particular.

Results: Projected final mean doses are summarized with respect to estimated near-maximal effective doses (Davis et al. 2003). With respect to CONSTA, the range of doses appears to be equivalent to only half of that available with the oral form.

Conclusions: This suggests that careful attention needs to be paid to patient selection. The current selection guidelines take in to account the censored dose range and it is suggested that this may influence those correctly assigned to therapy with this new medication form. It also suggests that the empirical finding that a proportion of patients require doses higher than the recommended maximum is consistent with known RISe distributions in clinical practice.

\section{Evolving trends in antipsychotic use in two major centers: 1998-2006}

\section{T Lambert ${ }^{1,2}$, T Callaly ${ }^{3}$, B Sing ${ }^{2}$}

'OPEN, OPEN/ORYGEN; ${ }^{2 T h e}$ University of Melbourne, Melbourne, Australia; and ${ }^{3}$ Barwon Health, Geelong, Australia

Background: In the past decade, there has been an evolution of psychiatric services, moving to a community model with the closing of mental hospital beds by 2000. During this period, new, arguably more efficient antipsychotics have been introduced. There are no longitudinal studies available of antipsychotic use based on individual subject case audits. This paper presents a pharmacoepidemiological perspective on this issue, comparing two large mental health service entities subject to the same statewide 'Framework' document for service provision and yet with unique prescribing philosophies.

Methods: Using a purpose-built database, prescribing information, sociodemographics and legal status data were collected from Australia, New Zealand and Asia. Data were acquired from community-treated patients or out patients. Data for two major centers in Victoria, Geelong and Northwestern MH network, were abstracted and analyzed with respect to their comparative usage trends. This analysis is performed for those with schizophrenia and those with bipolar disorder.

Results: The data indicate that although both centers evolved their prescribing as deinstitutionalization progressed, their disparate prescribing patterns 